\title{
Aortic surgery symposium 2010 discussions-Session I: Aortic root I and session II: Aortic root II
}

\author{
Moderator: Nicholas T. Kouchoukos, Missouri Baptist Medical Center, St Louis, Mo.
}

Panelists: Dianna M. Milewicz, MD, University of Texas Medical School at Houston, Houston, Tex; John Elefteriades, MD, Yale University School of Medicine, New Haven, Conn; Lars G. Svensson, MD, Cleveland Clinic, Cleveland, Ohio; Tirone E. David, MD, University of Toronto, Toronto, Ontario, Canada; Gebrine El Khoury, MD, St Luc Hospital, Bruxelles, Belgium; Ruggero De Paulis, MD, European Hospital, Rome, Italy; Duke E. Cameron, MD, Johns Hopkins Hospital, Baltimore, Md; Emmanuel Lansac, MD, Foch Hospital, Suresnes, France; Hans-Joachim Schaefers, MD, University Hospital of Saarland, Homburg/Saar, Germany; Thoralf M. Sundt III, MD, Mayo Clinic, Rochester, Minn.

\section{Discussion}

Dr Nicholas T. Kouchoukos (St Louis, Mo). I would like to direct a few questions to the presenters and then open up the panel to questions from the audience.

Dr Milewicz presented some fascinating data about the genetic implications of many of these aortic diseases, and she also emphasized the importance of surveying first-degree relatives. This raises the question of how we evaluate these patients. Obviously, imaging is a very important way of doing this, yet we are seeing considerable criticism being leveled at some of the imaging techniques we are using, principally computed tomographic (CT) scanning. Dianna, can you tell us what the best way is to evaluate these patients and what the safest way is?

Dr Dianna M. Milewicz (Houston, Tex). We do not have a clear answer for that. I think echocardiography is fine, particularly in families in which you know the aneurysm is occurring in the sinuses of Valsalva. I think it may be okay for ascending lesions if on the echocardiogram you get good imaging of the ascending aorta. If you do not, then you do have to go to CT scanning or magnetic resonance angiography.

Dr Kouchoukos. If you are not going to do an echocardiogram, if you are going to use another type of imaging, what about the issue of radiation exposure in patients who have to have sequential studies?

Dr Milewicz. That is a problem and I do not have an answer for that, other than you can use magnetic resonance angiography instead of CT.

Presented at Aortic Surgery Symposium 2010, New York, NY, Thursday, April 29, 2010.

Disclosures: Dianna M. Milewicz is a consultant for Marfan Foundation and receives grant/research support from Genentech, National Institutes of Health, and Doris Duke. John Elefteriades receives grant/research support from the National Science Foundation and Celera Diagnostics, and is a consultant for Medtronic, Inc, and Baxter. Tirone E. David is a member of the speakers bureau for Medtronic, Inc, St. Jude Medical, and Edwards Lifesciences. Ruggero De Paulis receives royalties from Vascutek Terumo. Emmanuel Lansac is a consultant for Coroneo. Thoralf $\mathrm{M}$. Sundt III receives grant/research support from Atricure, Inc, Boehringer Ingelheim, Bolton Medical, Edwards Lifesciences, Jarvik Heart, Inc, Sorin Group/Crabomedics, St. Jude Medical, Throatec Corporation, Ventracor, Inc, and Gore \& Associates, Inc. Nicholas T. Kouchoukos, Lars G. Svensson, Gebrine El Khoury, Duke E. Cameron, and Hans-Joachim Schaefers have nothing to disclose with regard to commercial support.

Address for reprints: Eva B. Griepp, MD, Department of Cardiothoracic Surgery, Mount Sinai School of Medicine, New York, NY 10029 (E-mail: ebgriepp@aol.com).

J Thorac Cardiovasc Surg 2010;140:S45-51

$0022-5223 / \$ 36.00$

Copyright (c) 2010 by The American Association for Thoracic Surgery

doi:10.1016/j.jtcvs.2010.07.052
Dr Kouchoukos. Dr Elefteriades presented some very good data that he has been accumulating over a number of years regarding the natural history of aortic aneurysms and the size limits for recommending surgery. John, you did not mention anything about the difference between patients with chronic dissection and patients with degenerative aneurysms of the descending thoracic aorta. Are the numbers equivalent? Should we be operating on smallerdiameter dissected aortas?

Dr John Elefteriades (New Haven, Conn). I do not have any data on that. The data that we have are similar to what has been shown by others: that the natural history is abysmal once the dissection has occurred. The survival curve is much lower. We have never separated out our chronic dissections from our degenerative aneurysms because the numbers are relatively small.

Dr Kouchoukos. I recall that Dr Griepp's group has shown that perhaps the risk of rupture is a little bit higher in patients with chronic dissection as compared with those with degenerative disease. Should we operate earlier than $6.5 \mathrm{~cm}$ in the descending aorta and $5.5 \mathrm{~cm}$ in the ascending aorta in patients who have chronic dissection in the absence of Marfan syndrome?

Dr Thoralf M. Sundt (Rochester, Minn). My practice is to be more aggressive in patients with a dissection, but I do not have data to support it.

Dr Kouchoukos. Do you have any numbers that you use for an aneurysm of the descending aorta, or a chronic dissection, as to when you would consider open—not endovascular—intervention?

Dr Sundt. For intervention in a chronic dissection, I would drop the criterion by about a centimeter. The other factor I would consider is the age of the patient. Craig Miller has talked about this: what you do with the young patient with severe hypertension whom you just believe is going to be a bad actor. I oftentimes bring such patients back much more quickly for a follow-up scan. Frequently, they demonstrate remarkable enlargement. Therefore, I think there is an age effect as well, and we probably should be more aggressive in younger patients.

Dr Kouchoukos. Let's move on to the valve-sparing procedures. Dr David showed us his results with his David V procedure and also indicated that he had done a fair number of procedures with a straight tube graft. Is there really any difference in the long-term outcomes between the two in your experience?

Dr Tirone E. David (Toronto, Ontario, Canada). It is an unfair comparison, because until about 1998 I had done only straight tubes. Therefore, the longest follow-up is with a straight graft. We have the lowest incidence of reoperation in that group. We started tailoring the grafts, and at the same time we started tailoring 
the cusps, and we started treating patients who were in more serious condition. That is why all these retrospective reviews are difficult to use in weighing one technique against another. The answer is that in our hands a straight tube or a tube with a Valsalva has not yet made a difference after 20 years of experience.

Dr Kouchoukos. Dr De Paulis showed some data that the outcomes were better in the patients who were operated on with $1+$ aortic regurgitation in comparison with those who had greater degrees of regurgitation. Has that been true in your experience as well?

Dr David. No, not at all. Cusp repair has no effect on late outcome. However, there is an intrinsic bias there. As the surgeon, it is my inspection that determines what is a good cusp or a bad cusp. To have all 3 cusps damaged is not uncommon. If you are at a referral center, you are getting younger and younger patients who come to you to have a valve-sparing procedure, not to have a Bentall procedure. So we repair the valves, and thus far, cusp repair has not increased the risk of a bad outcome. I think Joachim has had the same experience, and Gebrine, whom I trained, has had the same experience: cusp repair does not increase aortic insufficiency.

Dr Ruggero De Paulis (Rome, Italy). There are two different situations with regard to cusp prolapse: sometimes cusp prolapse is unrecognized by the surgeon, but sometimes it can be induced by the procedure, especially because the patient's geometry is being altered, at least a little bit. That is why we may have some differences in results. I do not think we can do anything about this. It is difficult to judge which procedure is best if you start with a different patient.

Of course, the best way to judge two different procedures is to start with a perfect leaflet. Early in the process of dilatation of the root, we can probably find a good leaflet. I think if you want to increase the chance of having a perfect reconstruction, you should operate a little before you get significant aortic insufficiency, even though it is difficult to find a relationship between the degree of preoperative aortic insufficiency and the optimal result of the operation: often this is because the surgeon used other maneuvers in addition to simple root reimplantation.

Dr Kouchoukos. We have been presented with superb results from all of the surgeons who have discussed the valve-sparing procedures: extremely low mortality rates and extremely low reoperation rates. Excluding patients with Marfan syndrome, does this mean that we should be repairing these valves at an earlier time, even in the absence of any aortic regurgitation? If so, where do we draw the line?

Dr Hans-Joachim Schaefers (Homburg/Saar, Germany). I have a somewhat different belief. I like Thor's presentation of the denominator problem. The tool we have is effective but not harmless. If we lower the threshold for operation, we will include many patients who currently are being treated conservatively, and at this time we have no calculations as to whether we are actually doing something good or whether, looking at the whole population, we are creating more harm than good. I think we will really have to carefully consider that possibility. I am often confronted with that question by referring cardiologists, who point out that we can repair the valve, and then ask whether we should proceed along lines similar to what we do with the mitral valve, where the severity of mitral regurgitation is sufficient indication for surgery in guidelines both here on this continent and in Europe. However, with the mitral valve, we have a track record of 30 years. In aortic valve repair, especially when the cusp aspect is involved, we are looking at 10 or 15 years, and I think it is simply too early to become more aggressive.

Dr David. I think if the patient needs an operation, the operation should be done. But first you must find out who needs an operation. The natural history, as correctly presented by both John and Thor, is not that bad. The progression is steady. Size does not matter anymore. Half the dissections occur in patients with a normal-sized aorta. So are you going to go out and replace every aorta? I think the traditional indications for surgery should prevail.

Dr Kouchoukos. Thor, you raised some important and controversial issues that are relevant to the present discussion. What about a patient who has a bicuspid valve that is functioning normally and has a dilated ascending aorta? When do you make a recommendation to replace that aorta?

Dr Sundt. I have a 50:50 rule: if the aorta is $50 \mathrm{~mm}$ and the patient is 50 years of age, I throw up my hands and advocate replacing the aorta. But much lower than $50 \mathrm{~mm}$, I am hard-pressed to find an argument to replace that aorta.

I think that Tirone is right. The problem is that size is not a good predictor. We need the genetics. There are two sides of this equation. Dissection is a structural failure of the aorta, and it is related to the mechanical stresses and the material properties of the aorta. The stresses on the aorta are determined by the diameter and by the blood pressure. We know that patients with hypertension are at greater risk, and we can control their blood pressure.

Then there is the issue of the material properties, so smoking is a bad idea. We are given some clues by the genetics about the material properties. Patients with Marfan syndrome, for example, have abnormal material properties and thus they are at a higher risk of structural failure. However, until we have a more sophisticated assessment of what those material properties are, we are stuck.

Dr Milewicz. I want to mention that although we started our genetic studies focusing on gene mutations that were similar to Marfan syndrome, more recently we have looked for genetic alterations that are there in the population but predict ascending aneurysms and dissection. We will be coming out with some of those variants over the next year, and that may help us in guiding determination and timing of surgery both for patients with bicuspid aortic valves and for those without.

Dr Kouchoukos. The genetic testing you mentioned is expensive and not readily available. How is that going to change in the future?

Dr Milewicz. Genetic testing is actually readily available. If you go to the GeneTests Web site (http://www.ncbi.nlm.nih.gov/sites/ GeneTests), you will find a list of commercial laboratories that test any of the genes that I talked about except for the kinase gene that we found just recently. And the cost is falling rapidly. Sequencing technologies are improving almost on a monthly basis, and I think that the cost of these tests will continue to drop.

Dr Kouchoukos. What about insurance coverage of these tests?

Dr Milewicz. That is another question. I think this is something that we have to push as we identify more genes: to make sure that all insurance covers genetic testing. About half the policies do currently: we can push the insurance companies and get another quarter to pay.

Dr Kouchoukos. Let's open up the discussion for questions. 
Dr Sundt. Can I ask Duke a question? In the setting of LoeysDietz syndrome, what extent of the operation we should perform? Do you replace the arch or not? You talked a little about the approach being the same, but how much aorta should we replace?

Dr Duke E. Cameron (Baltimore, $M d$ ). We do not know the answer to that. That is a discussion that is ongoing. Until now, we have just replaced the ascending aorta up to the crossclamp. We do not do a hemiarch, and we do not routinely use circulatory arrest. However, it is our impression that we are seeing many more Loeys-Dietz patients come back with dilated arches than we have seen with Marfan syndrome. That is a question that we will have to answer, but we have not changed our practice yet.

Dr A. Trento (Los Angeles, Calif). I am following a fair number of patients with bicuspid valve-with a normally functioning valve and a dilated aorta - and I see that the aorta really does not increase in size: they have an aorta of 4.5 or $4.7 \mathrm{~cm}$, and it stays at that level. Obviously, this is a small number, but I have the impression that medical therapy in these patients will go a long way. What is your impression?

Dr Sundt. You can tell from my presentation that I agree with you, but John has some data about it.

Dr Elefteriades. Yes, this is an indolent disease, not only for those with bicuspid valves, but also for those with other ascending aortic aneurysms. The rate of growth is very low, $1 \mathrm{~mm}$ per year, and I think your observation is right on the money. Those patients will eventually grow if you look at them over 5 to 10 years. We cannot really detect growth until 3 or 4 years have gone by, because we cannot measure $1 \mathrm{~mm}$ at a time.

Dr David. I have never looked at this prospectively in my practice, but I have the impression that an aorta of $47 \mathrm{~mm}$ in a 20-year-old patient does not behave the same as a $47-\mathrm{mm}$ aorta in a 50-year-old patient. In the younger patients the aorta dilates more along your rate, 0.1 to $0.2 \mathrm{~cm}$ per year. In the older ones, the aorta does not. It seems to be more stable. Don't you think age is a factor?

Dr Elefteriades. We know that the aorta grows a little bit with age, even if it is normal. We have not ever identified a slowing of growth with advancing age, but we could look at that: it would be easy to look at.

Dr Sundt. It makes good sense that if the aorta is already $48 \mathrm{~mm}$ at 25 years of age, the material properties are abnormal.

Dr Milewicz. Therefore, the younger the patient, the more likely there is an underlying genetic variance.

Dr David. It accelerates the disease of a patient. I do not think all dilatations are the same.

Dr T. Beaver (Gainesville, Fla). Wonderful data from Dr Milewicz. I was wondering whether she had any screening recommendations. I would appreciate it if Dr Elefteriades or Dr Sundt or someone else from the United States could tell us what their current practice is if someone comes in with an aortic dissection. Do you start talking to the family and recommending CT scans for siblings?

Dr Milewicz. Definitely, if there is a family history, we recommend screening. As I mentioned previously, an echocardiogram may be sufficient in many of the cases. For patients without a family history, we would certainly recommend screening if they are young, starting with the parents. If both of the parents are normal, we are less worried about the siblings having aortic disease. For the older patients, we really just do not know.
Dr Kouchoukos. What about patients with bicuspid aortic valves? Do you recommend screening of their first-degree relatives?

Dr Milewicz. Inasmuch as that can run in families, we certainly recommend screening if there is a history of aortic dissections. The studies have shown that those families can have aortic disease with bicuspid aortic valve, bicuspid aortic valve without aortic disease, or aortic disease and no bicuspid aortic valve. So you definitely screen if there is a family history and also if the patient is young.

Dr J. Greelish (Nashville, Tenn). There was a review a few years ago in Nature Clinical Practice by Drs Sundt and Davies that looked at the morphology of aortic dilation in bicuspid valve disease and described various types: the root may dilate, the ascending aorta may dilate, or an intermediate form may exist. When making the decision to do aortic replacement in a patient with bicuspid valve, is it always necessary to perform a root operation, or is a supracoronary graft sufficient?

Dr Schaefers. I think this is a very good question, but as Dr Sundt implied, at this time we have absolutely no data. Because of the scarcity of data and the fact that many of these roots remain stable over time, I think it is safe simply to leave the root as it is and just replace the supracoronary ascending aorta. The important question - the final question on my conclusion slide-is this: at what diameter should we go ahead and replace the root? At this time we have some indirect evidence that the $45 \mathrm{~mm}$ that came from the Toronto group may also be reasonable, but this is a gut feeling. It is evidence level $\mathrm{C}$ that $45 \mathrm{~mm}$ may be reasonable, but we have absolutely no calculations, no numbers.

Dr De Paulis. What do you do in the case in which one sinus takes up most of the diameter of the root? Often with a bicuspid valve, you have two sinuses that are smaller and one that is bigger. This is a different situation. Probably you have to replace that sinus.

Dr Schaefers. It is a different situation whatever you do, whether you replace one sinus or whether you do a whole root replacement. Again, we have no evidence; we have no numbers. All we know is that the Laplace law is applicable also to the root, and the Laplace law does not really take into consideration that one sinus is abnormally large. There is some indirect evidence if we look at the patch plasty of coarctation, the old approach of putting a Dacron patch in the area of the coarctation. This proved to be not so good in the long term, because many of these patients had aneurysms develop. Maybe it is not such a good idea to have an asymmetric operation, to have plastic on one side of a root and natural aortic tissue on the other. But, again, the evidence level is maybe D: very weak.

Dr Gebrine El Khoury (Bruxelles, Belgium). For bicuspid aortic valve there is also the diameter of the sinus to consider. Even if the diameter is acceptable, we have to assess the quality of the aortic wall, and in many patients with normal aortic root diameter, we can see that there is no longer any wall in some areas: it has disappeared; the aortic wall is very thin. In such a patient, in my experience, I replace the aortic root.

The second comment is that if you are operating on a regurgitant bicuspid aortic valve, I think one of the elements required to stabilize the aortic repair and to have long-term durability is to do something with the aortoventricular junction and the sinotubular junction. If I am repairing a regurgitant bicuspid aortic valve in a young patient, even if the aortic root is quite normal, I will do valve-sparing surgery and repair the aortic valve. 
Dr Sundt. Those patients with aortic regurgitation most often have a phenotype that demonstrates enlargement of the sinuses. One of the things that needs to be mentioned is that when you talk about doing a root replacement in someone with normalsized sinuses, they have undisplaced coronary arteries. It is technically harder, at least it is for me, to mobilize those coronary buttons and reimplant them. So the other side of the risk equation goes up. I think the risk of replacing a normal root is higher than the risk associated with replacing a dilated root.

Dr Emmanuel Lansac (Suresnes, France). I have just a quick comment on the size of the root for graft placement. In our practice, when the root is below $40 \mathrm{~mm}$, we will leave the root, and if there is an indication for only the root, we go for $50 \mathrm{~mm}$. If the indication is for the valve with a root above $40 \mathrm{~mm}$ - and most of the time if it is $45 \mathrm{~mm}$-we will replace it. If it is a young patient and if the coronary ostia are high—when the root is above $40 \mathrm{~mm}$ - I think it is good to replace it and treat, as Gebrine El Khoury said, the annular base dilatation at the same time.

Dr C. Van Doorn (Aarhus, Denmark). As Dr Cameron showed very convincingly for the patients with Marfan syndrome, and as was also shown by the other speakers, the remaining native aorta is the major source of mortality and morbidity at follow-up. Could the panel please comment on blood pressure management after surgery, such as the use of beta-blockers and angiotensin-converting enzyme inhibitors, and also, in particular, the use of losartan?

Dr Cameron. I would venture that most people on this panel are probably not involved in the long-term management of blood pressure. The short answer is that I think we try to maintain aggressive control of the blood pressure, certainly keeping systolic less than $120 \mathrm{~mm} \mathrm{Hg}$ and pushing beta-blockers to the point that they are just tolerated. Increasingly in our institution, I think because of the interest in losartan and the way it addresses what we think is the pathogenesis of some of these aneurysms, virtually all of our patients are receiving losartan as well as a beta-blocker.

Dr Kouchoukos. Duke, do you have any data about the benefits of the treatment in the losartan group?

Dr Cameron. No.

Dr Milewicz. The interim analysis for the losartan trial in children with Marfan syndrome up to the age of 25 years should be available within the next 6 months, so we will know whether the trial continues or not.

Dr M. Janusz (Vancouver, British Columbia, Canada). If you have a patient with mild to moderate aortic stenosis and a $51-\mathrm{mm}$ ascending aorta, the valve, if left alone, would not represent much of a hazard to the patient for several years, but no one is going to leave it behind. Are they better to have a prosthesis for several extra years or to be followed up with an aorta that is bigger than the recommended guidelines?

Dr Sundt. How old is the patient?

Dr Janusz. Let's say 50 or 60.

Dr Sundt. The older the patient is, the closer you are to being in the clear for one of the options being a bioprosthetic valve with a low risk of reoperation. So the older the patient is, the more easily one could argue for a lower threshold for surgery. Why take the risk of dissection when you have a good chance that a bioprosthetic valve will be durable? It is tougher in a patient who is 50 .

Dr Janusz. It is not just a question of durability; it is the annual risk of endocarditis and thromboembolism. In that size range, 50 to
$55 \mathrm{~mm}$, is the hazard of carrying the valve greater than the annual risk of dissection? I have not seen any data on that.

Dr Schaefers. I am not aware of any published data. I see your dilemma, and I would feel the same. In case of doubt I would probably remember what Thor Sundt pointed out: we are not $100 \%$ certain whether a 51-mm ascending aorta in the presence of a bicuspid valve is all that deadly. I would prefer to monitor the patient carefully, making sure he or she has good blood pressure control and so forth.

The guidelines actually open a rational window, in cases of moderate aortic stenosis, for looking at the degree of calcification. If calcification is very severe, the likelihood of that patient needing valve surgery within the next couple of years is very high, which would then give you a possible rationale for earlier surgery. I think this decision - in the absence of good data for this specific situationhas to be individualized.

Dr Kouchoukos. Thor alluded to this problem: we have all seen patients who have had an aneurysm that is discovered on an incidental CT examination. Body scans that were popular a few years ago uncovered a lot of patients who had aneurysms. Some of these patients arrive in your office totally terrified either by their own reading on the Internet or by what they have been told by their physicians. Some of them demand to have their aortas removed. How do we deal with this dilemma?

Dr Sundt. I think we need to quit scaring people to death: that is part of my plea. The woman that I discussed with you was seen by Heidi Connolly, one of our cardiologists who specializes in cardiovascular disease in women in pregnancy. Heidi recommended that she could have a pregnancy with careful control of her blood pressure; I said that she could have a pregnancy. However, she was so terrified by what she had been told before that she went home and had a composite root replacement and had her tubes tied. I think that is a tragedy. Once the seed has been planted that someone has a time bomb ticking in her chest, it is very hard to reassure her. I think that we need to be responsible about what we tell the public.

Dr R. Calhoun (Sacramento, Calif). Moving to a slightly less elective scenario, I am curious about the panel's philosophy in patients who have a type A dissection, but type A in the sense that they may have a pericardial effusion. They may have early tamponade. They need something urgently done. However, the tear itself is in the arch or perhaps even distal to the subclavian. What is the philosophy? Are we there simply to deal with the ascending aorta and save the patient's life, or should people be chasing these tears even if it means doing a complex arch reconstruction at 3 in the morning?

Dr Schaefers. I do not think there is a quick and simple answer to this difficult question. I would suggest discussing this after the arch session.

Dr Kouchoukos. I think we will leave it for later.

Dr H. Sherif (Newark, Del). In cases of bicuspid aortic valve, the dilatation in the ascending aorta is often eccentric at the greater curvature. It is not the usual fusiform shape of many other aneurysms. What do you think about segmental patterns of aortic dilatation in specific disease entities and how they affect management of that particular situation?

Dr Schaefers. We know that we do not know. It is a very good thought. Actually, over the past $1 \frac{1 / 2}{2}$ years, we have selected samples of different areas of the ascending aortic wall to look into that 
question. The key question is whether this is a consequence of hemodynamics, of turbulence directed toward the outer curvature, or whether there is a regionalized specific phenomenon in the aortic wall. So far, all the information we have is global and does not differentiate specific areas of the aortic wall. I think there is no good answer to your question, but it is a good question.

Dr Kouchoukos. I believe there was an abstract presented at the American Heart Association meeting describing histochemical analyses in different parts of the ascending aorta. The authors did identify differences between the greater curvature and the lesser curvature. Dianna, do you have any thoughts about that?

Dr Milewicz. My argument would be that there would be more changes with the hemodynamic stresses on one side of the aorta, which is what you would expect to see, rather than that the response is an attempt to repair what the wall is sensing is a vascular injury.

Dr C. Miller (Stanford, Calif). I am delighted by the blunt honesty of this panel. Maybe as we all get older, we get a little more mature and wise, but I have never heard so many distinguished experts say "I don't know." I really applaud all of you.

Duke, did I hear you say that Hal Dietz has convinced you to push the beta-blockers to the point of tolerance in addition to losartan in the bicuspid valve patients?

Dr Cameron. I do not think I said that, but I think that is his practice.

Dr Miller. Does he perhaps think it is the same disease even though it does not involve fibrillin-1 or transforming growth factor beta perhaps?

Dr Cameron. I am sure he would not say it is the same disease, but I think he believes there is a lot of overlap.

Dr Miller. Dianna?

Dr Milewicz. We do not know.

Dr Miller. I love that. We were talking about replacing a valve and leaving some generous sinuses, or Gerbine says we are saving a leaking bicuspid valve in a young patient and the sinuses are not that big. I agree with Gerbine $100 \%$. It is very uncommon not to have annular dilatation be an important component of the mechanism of leak in any type of bicuspid aortic valve, whether it be Sievers 0 or Sievers 1 , and if you do not address the annular dilatation, you are probably not going to have a very durable valve repair. One of the panelists also said to look at the quality of the wall of the aortic root: that was Gerbine, too. Who cares what size it is if it is transparent? You had better replace those sinuses even if it is a Fazel cluster II aortopathy in which the sinuses are of relatively normal size.

Dr J. Bachet (Abu Dhabi, United Arab Emirates). I would like to ask a very simple question of the experts of the valve-sparing procedure here on the panel. They gave us a lot of data, but there is one figure that I never find in the papers or presentations. How many patients, in their experience, go into the operating theater with the intention of having a valve-sparing procedure and come out with a valve? In my small experience of 75 aortic valve-sparing procedures, there were, in addition, 5 patients in whom I tried to preserve the valve, but I had to replace it because my result was poor. These data are never provided. It would be interesting to know the rate of intraoperative failures in the experts' experience.

Dr Kouchoukos. That is an excellent question.

Dr Cameron. We have written on that. I think I actually said at this meeting a couple of times that about $10 \%$ and in some years almost $20 \%$ of the patients who come to the operating room for a valve-sparing procedure leave with a more traditional composite graft. You can set your threshold wherever you want as to what you think is a salvageable valve, and a lot of it has to do with the age of the patient. We will bend over backward to save the valve of a 10-year-old child, but in a 70-year-old patient with a lot of fenestrations who comes with a fair amount of aortic insufficiency to start with, we are not going to work as hard. It will also depend on the nature of your referral practice. We have a lot of very young patients, but even with that, $10 \%$ to $20 \%$ will have the valve replaced. We will take into consideration age, the number of fenestrations, and the amount of aortic regurgitation preoperatively.

Dr De Paulis. I think it would be around $20 \%$ before actually looking at the valve intraoperatively and about $10 \%$ after looking at the valve. This is especially likely in those cases in which, even after looking at the valve, you know you should not do it, but you give it a try because the patient is motivated.

Dr David. I am the oddball here. My figure is close to $0 \%$. However, I do not take any patient before seeing a transesophageal echocardiogram. As with mitral valve repair, I do not replace a mitral valve that I take on to repair. If I tell the patient I am going to repair it, it is because the transesophageal echocardiogram showed me that the leaflets are reparable. Aortic valve sparing is the same thing. If you have no leaflets, do not try to repair or you end up with more pericardial patches than native tissue. Transesophageal echocardiography is the gold standard in my experience to determine whether the aortic valve is salvageable or not. To be quite honest, in the past 10 years I do not remember one patient accepted for valve sparing who went home with a Bentall procedure, except perhaps in bicuspid aortic valve. I told the patient that one cusp did not look too good and that he might end up with a Bentall procedure. But with a tricuspid aortic valve, the rate is very close to $0 \%$.

De El Khoury. I agree completely. There are two determinants for reparability of the valve. The first is the quality of the leaflets, and it will be very well assessed by the echocardiogram: 3-dimensional, transesophageal, or whatever. The second factor is how aggressive or experienced is the cardiac surgeon. In my experience, I can tell you that this patient, when he comes to the operating room, has a $100 \%$ chance to have the valve repaired or $100 \%$ likelihood to have it be replaced. It is really very easy now to assess the reparability of the aortic valve or the feasibility of valve-sparing surgery provided we have a good assessment of the quality of the leaflets beforehand.

Dr Kouchoukos. Tirone, what on the echocardiogram tells you that you are not going to be able to repair the valve?

Dr David. If the leaflets are calcified, if the free margin is rolled in, and if it is very echogenic. If all 3 leaflets are very echogenic at the free margin, I tell the patient a repair will be inadequate. If the height of the leaflet (you measure the height when the leaflet is open) is $10 \mathrm{~mm}$, it is reparable, but we are not offering a repair. That is perhaps why my results are so good. I am cherry-picking. I am repairing those that can be repaired and trashing those in which repair might be marginal. We consider the height of the leaflet, how echogenic the leaflets are, and their thickness. A commissural regurgitation in our hands almost invariably means fenestration, but fenestration, as I mentioned earlier, can be repaired with polytetrafluoroethylene (Gore-Tex; W. L. Gore \& Associates, Inc, Flagstaff, Ariz). 
Dr Kouchoukos. Some can and some cannot, correct?

Dr David. Some cannot, but if there is massive regurgitation through a commissural area, it is no longer a fenestration. The leaflet has become detached. In those cases, I do not tell the patient I am going to repair. Once I accept a patient with a transesophageal echocardiogram, I think that happens rarely; I only remember one instance in the past decade. However, I tell many patients that they should not have a repair: a Bentall procedure with valve sparing is a bad operation if the echocardiogram showed severely damaged cusps.

Dr Lansac. Yes. Thank you, Jean, for your question. Interestingly, there are 2 points. There are candidates for valve sparing who will not have it in the operating room, and actually, in my experience, it is more likely elderly patients with fenestrations. However, I think it is also interesting to know how many patients come to the operating room to have a valve-sparing procedure and then are converted.

Dr Bachet. That was my question.

Dr Lansac. That is the way I understood it, and that was interesting. We started a review of the literature and of our initial experience with the isolated remodeling technique, and we had a $30 \%$ failure rate in the operating room: remodeling cases that were converted to aortic valve replacement. From that point came the idea of combining the remodeling technique with a subvalvular annuloplasty. In the review that I presented to you, of more than $187 \mathrm{pa}$ tients, the rate of conversion using remodeling alone dropped to less than $3 \%$ when we carried out the annuloplasty. What will reduce even further the rate of conversion in the operating room-and I agree that this should be published in every report-is the standardized management of the cusp, especially using the effective height concept developed by Joachim Schaefers. This concept may help increase the reproducibility of the technique and help reduce failure in the operating room.

Dr Bachet. Tirone and Gebrine say that they never had a technical failure. Do you mind if I remain a little skeptical?

Dr David. No, I did not say that. I said that in the past decade, patients whom I said would have their aortic valve repaired had the repair. If you read all my articles, I am very open in saying that my very first reimplantation worked well, and that the second one failed in the operating room, and on the second day, the valve came out. This is a different story. You asked me, if you look at a valve, can you say whether it can be repaired or not? I consider myself expert enough to say. And so far in the past 200 cases, I have not let the patient down. So it is possible to detect a patient suitable for valve sparing before surgery.

Dr Schaefers. I would agree that valve sparing can be done in $95 \%$ or so. I do not want to go into a big philosophical discussion but rather to volunteer a couple of numbers. Intraoperative conversion after a failed repair is $1 \%$.

Intraoperative conversion after trying a repair that does not look so good-going for replacement rather than testing the valve by transesophageal echocardiography-is in the range of $2 \%$. In patients in whom I find a valve that will not last very long, considering patient age and so forth, replacement is probably somewhere in the range of $5 \%$ to $10 \%$.

Dr El Khoury. In the beginning of my experience, I had to repair the valve again in $5 \%$ to $7 \%$, but now we are really close to $0 \%$. We can achieve an immediate repair; it is predictable. Now the discussion is about durability, and I agree that this is something else. But for the immediate achievement of a successful repair, I think we can accept a $98 \%$ to $99 \%$ reparability rate.

Dr Lansac. I agree that with experience the rate of conversion is getting lower, but the main problem is the lack of standardization of the technique. To further lower the rate of failure, we think it is very important to standardize this complex operation, breaking it down into very simple steps and standardizing every step in root and cusp management. The majority of us will not treat a lot of these patients: between 2 and 5 patients per year. Even if there are experienced centers that are very good, most patients, in real life, are operated on in the hospital nearest their home. So we need to have strict criteria to manage the root and to deal with the technique to have reproducible results in the operating room and to reduce the failure rate. The lack of standardization is the main limitation to the widespread use of the valve-sparing procedure.

Dr M. Misfeld (Leipzig, Germany). I would like to know the panel's opinion regarding indications for performing an aortoplasty. Is there still any indication to do this and, if so, up to what size? Replacement of the ascending aorta increases the wall stress on the residual aorta, which may have an impact on its development of aneurysms.

Dr Elefteriades. Francis Robicsek is the remaining proponent of aortoplasty, and he wrote a nice article on that which came out about 5 years ago showing good results. I think most of the world has left that behind, because the added work of doing an aortic replacement is really very little beyond the work that would be required for an aortoplasty. In my own practice, I do it on rare occasions, but I reserve it for somebody who is perhaps very old, needs a ton of grafts, needs a valve, and we just want to do something to minimize the likelihood of an aortic event in the next few years.

Dr Kouchoukos. Do you wrap it, John, or are you comfortable just reducing the diameter?

Dr Elefteriades. We are comfortable just reducing the diameter. I worry about a wrap that does not go down to include the annulus the way the Florida sleeve does, because I think there is a lot of tension at the edge of that wrap.

Dr B. Koul (Lund, Sweden). I would like to take up the question of Dr Jean Bachet and extend it further. Drs El Khoury and Schaefers have taught us that when the coaptation height is less than 4 $\mathrm{mm}$, or the line of coaptation is near the annulus or subannular, the long-term failures are high. We saw today from Dr Schaefers' presentation that he is now more and more aggressive about raising the coaptation height to reach 8 or $10 \mathrm{~mm}$. How has this affected your repair success rate? Are you ending up with good coaptation but with cusps that do not meet in the center? How does it affect the intraoperative success rate?

Dr El Khoury. There is a difference between the optimal height and what we have. For the optimal height, in the normal aortic valve, the leaflet should be at the mid height of the sinuses. We found in our study that if the coaptation is at more than 4 or 5 $\mathrm{mm}$, the durability is acceptable. But I think the durability can be perfect if we achieve excellent coaptation. This gives me the occasion to make one comment. When operating on patients with a normally functioning aortic valve and with a root aneurysm, I think if your surgical techniques give you the same geometry of the valve without adding any leaflet maneuvers, you have an 
excellent surgical technique for sparing surgery. For years we have heard that intervention on the leaflet is more and more frequent. I think it is because we have not yet found the optimal, appropriate surgical technique. If I go from a normally functioning and normally configured aortic valve and root annulus, I end up with a normal configuration of the aortic leaflet without any intervention on the leaflet. This gives the impression or the feeling that one must have gotten the surgical technique exactly right. The second comment is that the level of coaptation is crucial for the durability of the aortic valve repair.

Dr Schaefers. A scientific answer: in a receiver operating characteristic analysis, an effective height of more than 8 or maybe $9 \mathrm{~mm}$ was a positive predictor of durability. This referred to the bicuspid valves, which we have studied best. Second, mentally, for me, measuring something — rather than eyeballing, questioning myself, reconsidering, and so forth-makes this operation more reproducible. Thor brought up an interesting question just after you mentioned yours. Can you bring the cusp up too high? Yes. If you shorten it too much, you will make it restrictive and you will induce restrictive aortic regurgitation. At this point, we have a tool that we can use, whereas before we had no tool. It is not ideal yet, but it allows us to guide the operation in a halfway predictable fashion.

Dr Kouchoukos. I thank all of the panelists for their excellent presentations and for the discussion. 